\title{
Ordered Ag and Cu Nanorod Arrays for Enhanced Raman Scattering Created via Guided Oblique Angle Deposition on Polymer
}

\author{
M. Keating ${ }^{1}$, S. Song ${ }^{2}$, G. Wei ${ }^{1,3}$, D. Graham ${ }^{4}$, Y. Chen ${ }^{1 *}$ and F. Placido ${ }^{2}$ \\ ${ }^{1}$ Photophysics Group, Centre for Molecular Nanometrology, Department of Physics, SUPA, \\ University of Strathclyde, John Anderson Building, 107 Rottenrow, Glasgow, G4 0NG, UK \\ ${ }^{2}$ Thin Film Centre, SUPA, University of the West of Scotland, Ayr, KA8 0SX, UK \\ ${ }^{3}$ Department of Physics, Beihang University, Beijing 100191, China \\ ${ }^{4}$ Centre for Molecular Nanometrology, WestCHEM, Department of Pure and Applied \\ Chemistry, University of Strathclyde, Glasgow, G1 1XL, UK
}

*corresponding author, tel: +44 (0)141 5483087 y.chen@strath.ac.uk

\begin{abstract}
We report the manufacture of ordered silver and copper nanorod arrays for surface- enhanced Raman scattering using oblique angle deposition (OAD) on pre-patterned polymer sheets. It was found that the patterned polymer substrate defined nucleation sites which guided subsequent growth of nanorods. Enhanced SERS intensities of the Raman probe molecule, trans-1,2-bis-(4-pyridyl) ethylene (BPE), were found for Ag arrays on polymer, up to about 10 times that of the Ag-silica control. The SERS response of Ag nanorod arrays of different structures was investigated alongside results obtained from discrete dipole approximation simulations. This revealed that narrow gaps between nanorods, formed by guided nucleation during $\mathrm{OAD}$, were responsible for this dramatic enhancement. Ordered $\mathrm{Cu}$ nanorod arrays were also successfully fabricated, producing a SERS intensity about three times that of $\mathrm{Cu}$ on
\end{abstract}


silicon for both BPE and another Raman probe - rhodamine B isothiocyanate (RBITC), highlighting the potential of this large-scale, low-cost SERS active substrate.

\section{Keywords}

\section{SERS, Metallic Films, OAD, Controlled Nucleation, Polymer}

\section{Introduction}

Surface-enhanced Raman spectroscopy (SERS) combines molecular fingerprint specificity with potential single-molecule sensitivity and so is an attractive tool in biological sensing and chemical analysis. To establish SERS as a routine analytical tool however, it is essential to produce powerful, reproducible SERS-active substrates in a cost-effective way. Tremendous efforts have been made in the last decade to develop various effective SERS substrates. Using numerous fabrication methods, a wide range of metallic nanostructures can be produced [13], DNA-conjugated nanoparticle clusters [4], nanoparticle arrays formed through molecular self-recognition [5] and nanoparticle self-assembly within a matrix [6, 7]. Alternatively, metallic films of various structures have also been created for use in SERS applications. In particular, aligned Ag nanorod arrays created by oblique angle deposition (OAD) have recently been demonstrated to be highly effective SERS substrates [8-11]. Producing strong SERS with high sensitivity, these arrays also show good reproducibility in signal strength and can be fabricated with substantial uniform areas for applications such as sensing. In addition, the OAD approach is relatively straightforward compared with other methods employed to generate nanostructured arrays, such as nanosphere lithography or electron beam lithography (EBL), and avoids the time-consuming, complex and expensive steps inherent in those methods [12]. Ag nanorods have also been applied successfully in 'real world' applications, for example, in the detection and differentiation of several human pathogens [13]. However, despite their obvious success, it has proved difficult to control the gap size and diameter of nanorods during $\mathrm{OAD}$ fabrication, hindering the optimisation of geometrical parameters 
which directly affect the SERS response [14].

The dimensions of nanorod arrays are initially influenced by nucleation, which is a random process on flat substrates, meaning nanorods created by OAD are randomly distributed. To obtain a high-order nanorod array via the suppression of random nucleation events during OAD, Liu et al. used two-dimensional Au nano-post arrays manufactured using EBL, where the posts acted as periodically arranged shadowing centres during Ag deposition. While a dependence of the SERS response on gap-size and diameter was found, the arrays produced were only semiordered. Moreover, the size of the arrays was very small $(50 \mu \mathrm{m} \times 50 \mu \mathrm{m})$ which is not suitable for sensing applications [14].

In this work, we demonstrate for the first time the application of polycarbonate sheet, prepatterned using nanoimprint lithography (NIL), as a large-area, highly uniform template to tightly control the nucleation process during $\mathrm{OAD}$ and in turn, the subsequent growth of ordered metallic nanorod arrays for SERS. Guided nucleation makes optimisation of SERS possible, based on template design which steers gap size and diameter towards the most favourable values.

\section{Experimental}

\section{Materials}

Silver and copper (99.99\%, Kurt J. Lesker and Company); trans-1,2-bis-(4-pyridyl) ethylene (BPE) (Sigma Aldrich assay 97\%); rhodamine B isothiocyanate (RBITC) (Sigma Aldrich); Polycarbonate sheets were prototypes supplied courtesy of MacDermid Autotype Ltd. 


\section{Nanoimprinted Polycarbonate}

Polycarbonate sheets (A4 size) with an inverted hemispherical structure in a closely-packed hexagonal arrangement (SEM image in fig.S1), fabricated using nanoimprint lithography were used as received. Nanoimprinted polymers have been employed previously in generating plasmonic structures [15-17] via the deposition of metallic layers on patterned polymer through sputtering. Flat polymer bases have also been used, in Ag nanorod OAD for example, to create flexible, large-area SERS substrates resistant to mechanical strain which could potentially be used as flexible SERS 'labels' [18]. Underlying polymer layers, patterned or flat, can generate highly reproducible and high-throughput SERS substrates at low cost. In this work the polymer serves as a seed template during OAD.

\section{Oblique Angle Deposition of Ag and Cu Nanorod Arrays}

Nanorod fabrication using OAD has been described in detail elsewhere [19, 20]. Briefly, polycarbonate substrates, cut to approximately $10 \times 13 \mathrm{~mm}^{2}$ and adhered to glass slides, were loaded for metal deposition, together with silicon, silica or glass substrates for comparison. A Satis e-beam evaporation system was used for thin film deposition, with the chamber vacuum maintained at about $1 \times 10^{-6}$ Torr. Deposition along the surface normal ( 0 degrees) resulted in conformal growth and an ordered Ag nanorod array as shown in fig. 1(a) (also large scale in fig.S2). 

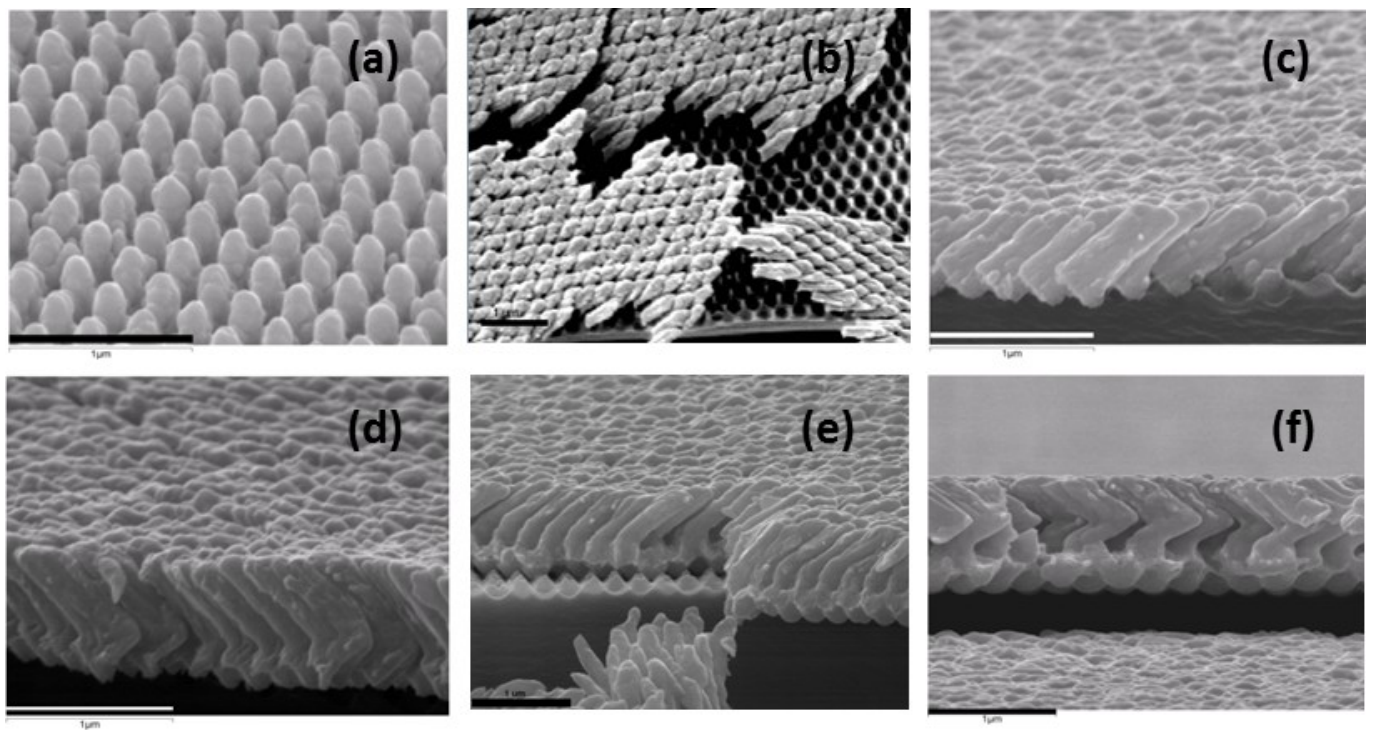

Figure 1. SEM images of Ag nanorod arrays on polymer films prepared under (a) $0^{0}$ deposition; (b) $85^{0}$ deposition (top view); (c) $85^{0}$ deposition (cross section); (d) $85^{0}:-85^{0}$ deposition; (e) $0^{0}: 85^{0}$ deposition and (f) $0^{0}: 85^{0}:-85^{0}$ deposition. The scale bar in each image represents $1 \mu \mathrm{m}$. Note: parts of arrays were lifted off when the samples were cut to create cross sections.

$\underline{\text { Before oblique angle deposition, a } 10 \mathrm{~nm} \text { layer of the respective metal (monitored by a quartz }}$ crystal microbalance) was deposited onto the substrates in the normal direction to increase nanorod adhesion. This thin metallic layer also serves to protect the polymer from laser illumination while simultaneously blocking the polymer background signal during a Raman measurement [21]. OAD was then performed at 85 degrees with a nominal growth rate of 0.2 $\mathrm{nm} \mathrm{s}^{-1}$. Micrographs of thus manufactured nanorod arrays were taken using a SEM (Hitachi S-4100). 


\section{SERS of Ag and Cu Nanorod Arrays}

Prior to SERS analysis, all $\mathrm{Ag}$ and $\mathrm{Cu}$ substrates were immersed overnight in an aqueous solution of trans-1,2-bis-(4-pyridyl) ethylene (BPE) $\left(10^{-5} \mathrm{M}\right.$ for $\mathrm{Ag}$ and $10^{-4} \mathrm{M}$ for $\mathrm{Cu}$ ), a common SERS probe, then dried in an $\mathrm{N}_{2}$ stream. 10 and 20 spectra were collected for $\mathrm{Ag}$ and $\mathrm{Cu}$ respectively, from the entire surface of each substrate (about $1 \mathrm{~cm}^{2}$ ) using a Renishaw Ramascope System 2000 with the $632.8 \mathrm{~nm}$ line of a helium-neon laser as the excitation source. Unfocussed output power at the sample was measured to be approximately 3.2 and $3.5 \mathrm{~mW}$, with collection times of $0.1 \mathrm{~s}$ and $30 \mathrm{~s}$ for $\mathrm{Ag}$ and $\mathrm{Cu}$ respectively. (More details are in the supplementary section).

SERS mapping of $\mathrm{Cu}$ substrates with $1.156 \times 10^{-5} \mathrm{M}(\mathrm{aq})$ rhodamine B ITC was conducted on a Witec Confocal Raman Microscope alpha300 R at $632.8 \mathrm{~nm}$ excitation, with a2.5s collection time and an unfocussed power at the sample of $24.3 \mathrm{~mW}$. Mapping of Cu polymer and Si arrays with $10^{-5} \mathrm{M}$ methanolic BPE was carried out on a DXR Raman Microscope (Thermo Scientific) using $780 \mathrm{~nm}$ excitation and a $50 \times$ objective with a $1 \mathrm{~s}$ collection time. (More details are in the supplementary section).

As regards optimal sample excitation, it has been reported previously that incident angle and polarization of the laser beam in respect of nanorod direction may influence SERS signal strength $[22,23]$. This however is not the subject of the current study, and in order to reduce the number of variables, all substrates were treated equally with a fixed angle of $0^{0}$ between the substrate plane and the E-vector, and also between the longitudinal planes of the nanorods and the E-vector. 


\section{Results and Discussion}

\section{Ag Nanorod Arrays}

Fig. 1(b) shows a SEM image of the nanorod array (note that parts of arrays have become dislocated from the underlying polymer during preparation for SEM imaging). Ordered arrangement of the array following a hexagonal polymer pattern, in contrast to random nanorods grown on a silicon surface (fig. S4), confirms that guided nucleation of nanorods has taken place. Figure 1 (c) shows a SEM image of a cross section of nanorod array on polymer. The diameter of the rods is about $187 \mathrm{~nm}$ and the length about $750 \mathrm{~nm}$. Subsequent depositions at 85 and -85 degrees (denoted by $85^{0}:-85^{0}$ ) resulted in a zigzag rod structure, as shown in fig. 1(d). Two other nanostructured arrays were produced by growing tilted $\left(85^{\circ}\right)$ and zigzag $\left(85^{0}:-85^{0}\right)$ nanorods on short vertical bases (denoted by $0^{0}: 85^{0}$ and $0^{0}: 85^{0}:-85^{0}$, and shown in fig 1(e) and (f) respectively).

Figure 2(a) shows typical SERS spectra of BPE on ordered $85^{\circ}:-85^{\circ}$ and $85^{\circ} \mathrm{Ag}$ nanorod arrays on polymer ( $\mathrm{B}$ and $\mathrm{C}$ respectively) in comparison with $85^{\circ} \mathrm{Ag}$ nanorod arrays on silica (A); the most prominent vibrational modes of BPE appear at 1200, 1607 and $1637 \mathrm{~cm}^{-1}$ corresponding to the $\mathrm{C}=\mathrm{C}$ stretching, aromatic ring stretching and in-plane ring modes respectively [7].

It is clear from figure 2 that both arrays on polymer generate a strong SERS signal, up to about 10 times that of the other polymer substrates and the silica control. In terms of reproducibility, the $85^{\circ}$ and $0: 85^{\circ}$ arrays have comparably low RSDs of $11.8 \%$ and $10.3 \%$ respectively, which are better than the $24.4 \%$ for $85^{\circ}$ arrays on silica, $34.0 \%$ on silicon and $33.4 \%$ for the $85^{0}:-85^{0}$ array on polymer. The $85^{\circ}$ array on polymer therefore surpasses all others when SERS intensity and reproducibility are considered in conjunction. Good 
reproducibility also qualifies the $85^{\circ}$ polymer array for SERS detection and identification purposes [24].
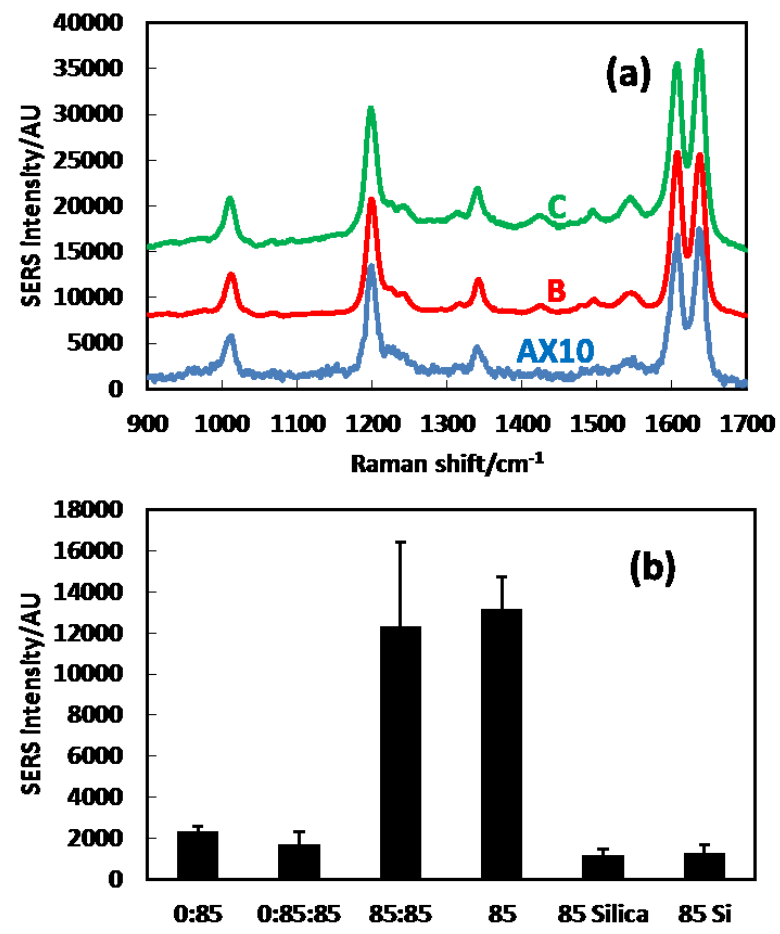

Figure 2(a) representative SERS spectra of BPE on Ag nanorods (excitation $632.8 \mathrm{~nm}$, acquisition time $0.1 \mathrm{~s}$ ): (A) $85^{\circ}$ silica control, (B) $85^{\circ}:-85^{\circ}$ polymer, (C) $85^{\circ}$ polymer, and (b) average intensity of $1200 \mathrm{~cm}^{-1}$ peak over 10 spectra from various Ag nanorod arrays as labelled.

All arrays, excepting $0^{0}$, have similar diameters and overall lengths as confirmed by SEM images. Their overall surface areas are comparable as shown in S5, meaning there is no significant correspondence between SERS signal strength and nanorod surface area in this case. 


\section{Simulations}

The nanorod array of $0^{0}$ deposition on polymer (figure 1(a)) produces a weak but definite SERS spectrum of BPE, 250 times weaker than does $85^{\circ}$ deposition on polymer, while in contrast, $0^{0}$ rods on silicon produce none at all (fig.S6). Our previous study found that the porosity of Ag film on planar silicon decreases dramatically when the deposition angle is reduced from $85^{\circ}$ to $75^{\circ}$, resulting in no discernible SERS of BPE at $75^{\circ}$ or less [19]. It is not surprising therefore that $0^{0}$ deposition on planar silicon generates no SERS. However, this cannot account for the substantial differences in signal strength between vertical nanorod arrays $\left(0^{0}\right)$, arrays supported by vertical bases $\left(0: 85^{0}, 0: 85:-85^{\circ}\right)$ and tilted arrays $\left(85^{\circ}\right.$ and 85 :$\left.85^{0}\right)$

To understand the SERS effect in terms of the electromagnetic (EM) enhancement mechanism, the local fields of infinite Ag nanorod arrays were simulated using the discrete dipole approximation (DDA) method, employing DDSCAT 7.2 code [25-28]. The dielectric constants of Ag were obtained from the experimental data of Johnson and Christy [29] without size corrections, as the size dependence can be neglected in our studies. The value of the interaction cut-off parameter $\gamma$ was taken to be 0.01 . Five target units, corresponding to $85^{0}, 85:-85^{0}, 0^{0}, 0: 85^{0}$ and $0: 85:-85^{0}$ arrays, were calculated using geometrical parameters shown in figure S7. The orientation of the oblique nanorods was chosen to be along the ydirection, and the tilted angle was set to 42 degrees. The upper oblique parts of the nanorods were modelled as tilted cylinders with a hemispherical cap at each end, to smooth sharp edges and so avoid the 'lightning rod effect'. Gaps between adjacent nanorods were $111 \mathrm{~nm}$ for $0^{0}$ arrays (estimated from SEM measurements) and $21 \mathrm{~nm}$ for other arrays. The total lengths of individual nanorods were all fixed to be $750 \mathrm{~nm}$ in order to investigate the structural effect on the near-field properties. The polarization of incident light was parallel to the y-direction. 

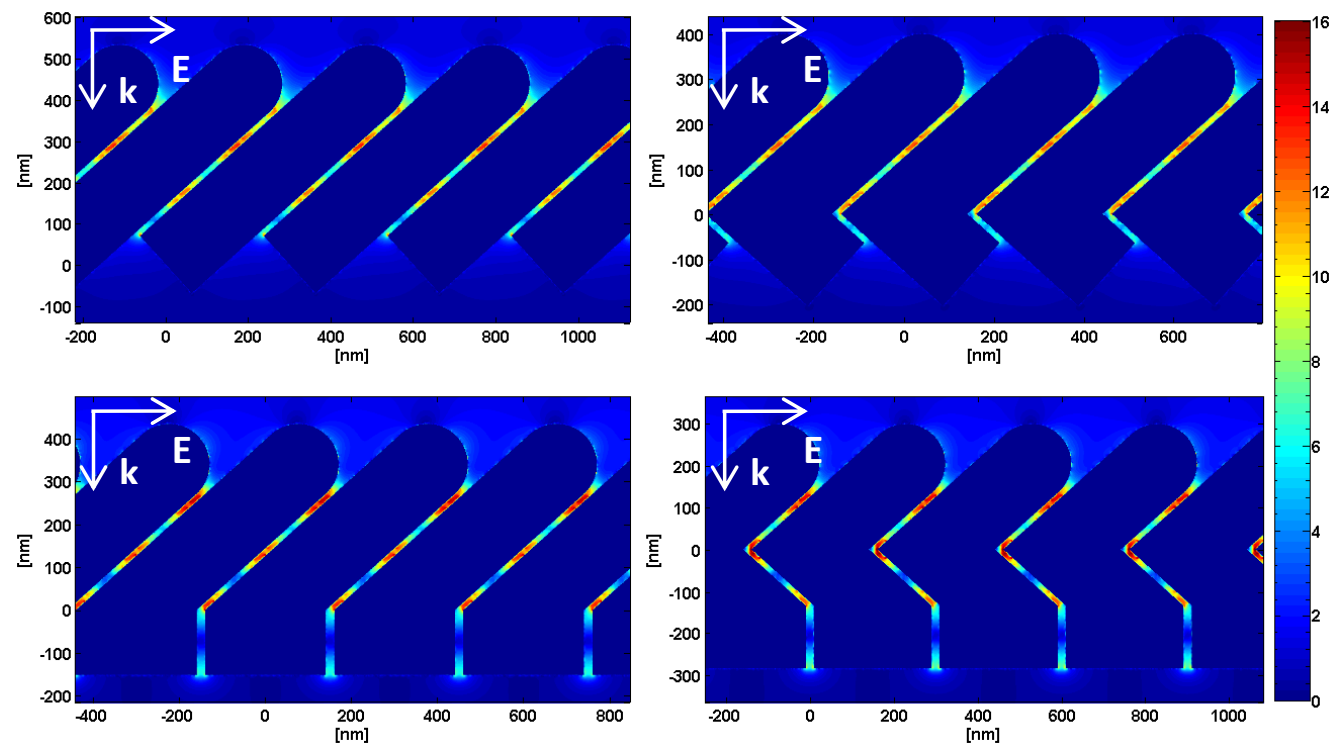

Figure 3. Magnitude of normalized electric field, $g=|E| /\left|E_{0}\right|$, where $E$ and $E_{0}$ are the local and

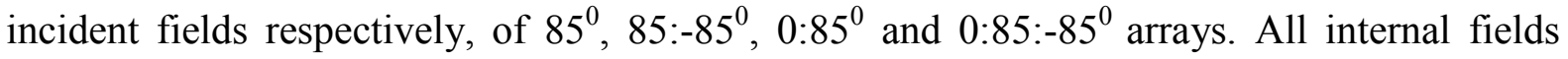
were set to zero for clarity.

Figure 3 shows the magnitude of the normalized electric field, when $85^{\circ}, 85:-85^{0}, 0: 85^{0}$ and 0:85:- $-85^{0}$ arrays are excited at $632.8 \mathrm{~nm} . g=|E| /\left|E_{0}\right|$, is the enhancement factor, where $E$ and $E_{0}$ are the local and incident fields respectively. Regions of strong EM enhancement (commonly known as 'hotspots') are found in the gaps between adjacent nanorods in all of the polymer arrays, with the exception of the $0^{0}$ array which produces by far the least enhancement due to a relatively large gap-size which promotes weaker coupling between neighbouring nanorods (fig. S8). At nanoparticle surfaces, the electromagnetic enhancement factor (EF) in SERS is proportional to $g^{4}$. To help explain our results, we calculated the average EF over the surface area of an $\mathrm{Ag}$ rod, $<\mathrm{EF}>=\int g^{4} d S / \int d S$ [30]. Note that the $\mathrm{g}^{4}$ value was calculated half a grid point away from each exposed cube surface. The average enhancement factors of $85^{\circ}$ and $85:-85^{\circ}$ arrays, 438 and 459 respectively, are comparable to each other and in line with experimental results. The slightly higher EF of the $85:-85^{\circ}$ array may be due to the sharp corners where the rods bend in the simulation structures. A much 
smaller EF of 29 was found for the $0^{0}$ array, also consistent with Raman measurements.

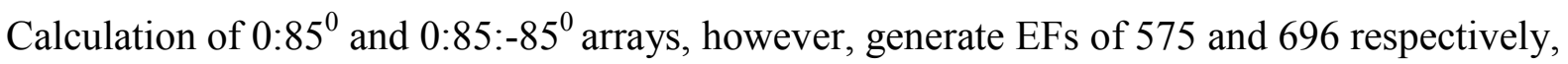
higher than those of the $85^{\circ}$ and $85:-85^{\circ}$ arrays. This inconsistency is due to the discrepancy in gap sizes between simulation structures and actual arrays. The magnitude of EM enhancement strongly depends on gap size, as shown in fig. S9. In the experiment, the diameter of real vertical rods, approximately $182 \mathrm{~nm}$ (resulting from conformal growth under $0^{0}$ deposition) was smaller than that used in the simulation $(279 \mathrm{~nm})$, resulting in larger gaps and a weaker EM field strength. Taking this into account, the calculations show a relatively weak EM enhancement from the $0: 85^{\circ}$ array compared with the $85^{\circ}$ array (fig. S10). Therefore, in this guided OAD approach the inverted hemispherical hexagonal pattern on polymer defines the periodic arrangement of the nanorod array and OAD produces tilted thick rods, resulting in narrow gaps between adjacent rods and in turn, a dramatic enhancement of the EM field. The dependence of rod size and associated plasmonic properties on polymer template dimensions needs further investigation.

For $85^{\circ}$ polymer arrays, BPE spectra taken under both 633 and $785 \mathrm{~nm}$ excitations show that the $\underline{\text { SERS intensity at } 633 \mathrm{~nm} \text { is about five times that at } 785 \mathrm{~nm} \text { (after corrections are made for the }}$ different scattering efficiencies of the two wavelengths) (fig. S11). S12 shows the magnitude of the normalized electric field of Ag nanorod arrays under different excitations simulated using DDA. The average enhancement factors were found to be 437 and 336 for excitations at $\underline{633 \mathrm{~nm} \text { and } 785 \mathrm{~nm} \text { respectively, which is in line with experimental results. The influence of }}$ excitation wavelength on SERS from Ag nanorod arrays depends on the geometric structures of arrays, as well as on the nature of molecules. Further investigation is required to reveal the mechanism. 


\section{Cu nanorods}

A number of metals have been employed successfully in SERS, the most common being silver, gold and copper [16]. Although the SERS signal is generally weaker when it is employed, copper is nonetheless highly attractive as a SERS metal, as it has a scrap value much less than that of either silver or gold. Recently, great efforts have been made to construct $\mathrm{Cu}$ based SERS-active platforms, including for example, nanowires, nanoparticles and hollow copper microcages [31-34]. Zhao et al. synthesised SERS-active vertical $\mathrm{Cu}$ nanorods using potentiostatic electrodeposition inside nanochannels of porous anodic alumina membrane (PAAM) [35], while SERS-active $\mathrm{Cu}$ nanorod arrays were created via OAD on glass slides by Kahn et al [36].

Given the effectiveness of Ag polymer substrates, we fabricated copper nanorod arrays on polymer using this guided OAD approach. An ordered array was created, as shown in figure 4(a). Substrates were immersed overnight in aqueous BPE $\left(10^{-4} \mathrm{M}\right)$ and interrogated using $633 \mathrm{~nm}$ excitation. Figure 4(b) shows typical SERS spectra of BPE for $\mathrm{Cu}$ nanorod arrays of

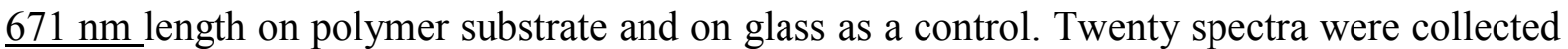
from each substrate. The array on polymer produces a SERS signal strength about twice that of the Cu-glass control as seen in $4(\mathrm{c})$ and the substrates have RSDs of $15.6 \%$ and $20.2 \%$ respectively. 

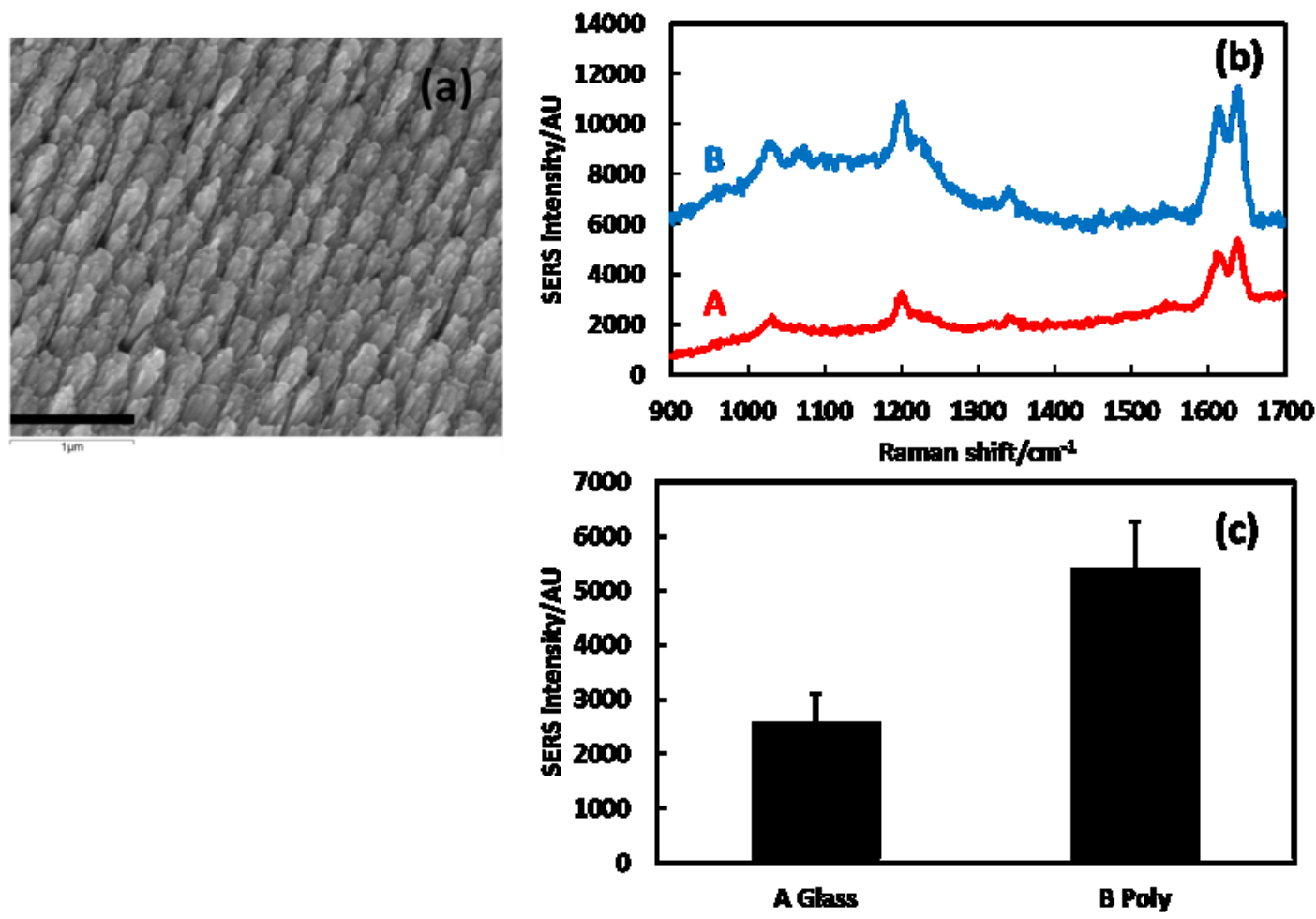

Figure 4 (a) SEM image of $671 \mathrm{~nm} \mathrm{Cu}$ nanorod arrays created under $85^{\circ}$ deposition on polymer showing ordered arrangement; (b) representative SERS spectra of BPE on: (A) Cuglass (B) Cu-polymer; (c) average intensity of $1636 \mathrm{~cm}^{-1}$ peak over 20 spectra from $\mathrm{Cu}$ nanorod arrays as labelled.

The effectiveness of $\mathrm{Cu}$ nanorod arrays on polymer substrates was further confirmed by SERS mapping using BPE as shown in figure 5. $2 \mu$ l of BPE in methanol $\left(10^{-5} \mathrm{M}\right)$ were placed on $\mathrm{Cu}-\mathrm{Si}$ and $\mathrm{Cu}$-polymer substrates and allowed to dry before SERS analysis. Each map corresponds to an area on the substrate of $90 \times 140 \mu \mathrm{m}^{2}$, with the $1636 \mathrm{~cm}^{-1}$ peak height used and 150 spectra collected. Mapping results averaged from 750 spectra from each substrate indicate that the $\mathrm{Cu}$ array on polymer (CuPoly) generates an average SERS intensity about 3 times that of the $\mathrm{Cu}$ silicon substrate $(\mathrm{CuSi})$. CuPoly shows good reproducibility at $23.9 \%$, which is better than that of $\mathrm{CuSi}(32.5 \%)$ 

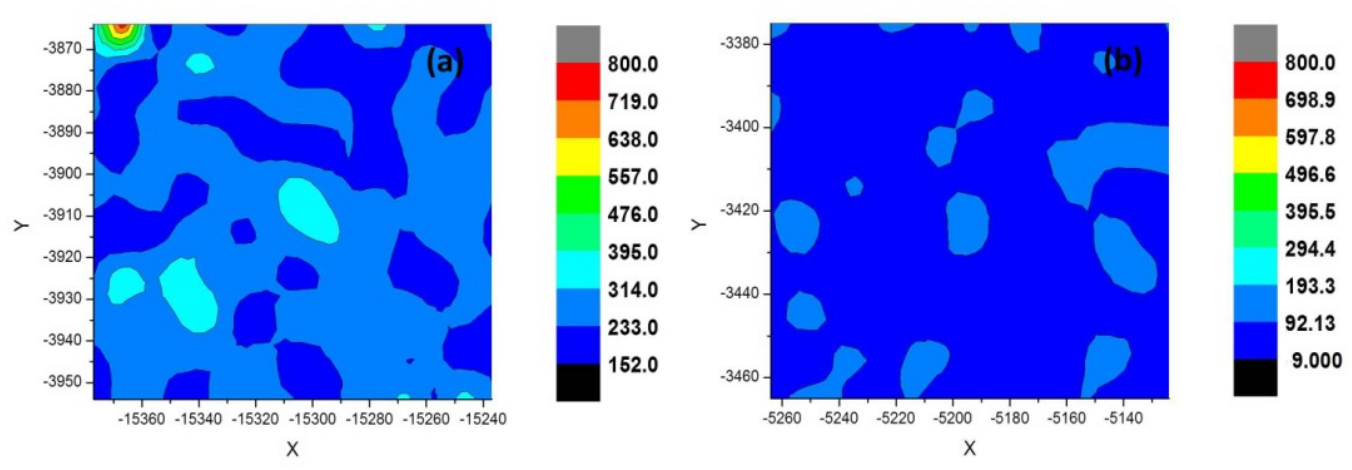

Figure 5 SERS maps of BPE $\left(10^{-5} \mathrm{M}\right)$ on (a) CuPoly and (b) CuSi. Each map corresponds to an area on the substrate of $90 \times 140 \mu \mathrm{m}^{2}$, with the height of the $1636 \mathrm{~cm}^{-1}$ peak being used and 150 spectra collected.
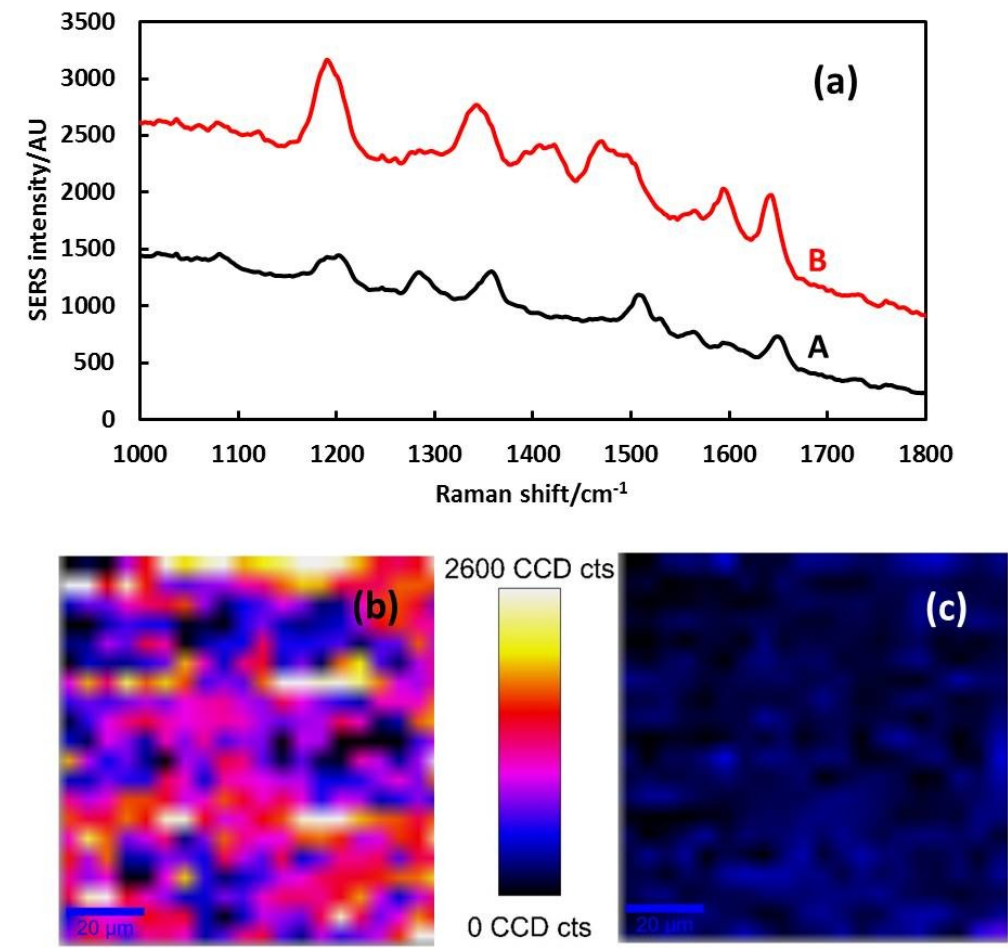

Figure 6 (a) SERS spectra of RBITC on (A) CuSi substrate and (B) CuPoly; (b) SERS mapping (from 400 spectra) of RBITC on CuPoly and on (c) CuSi substrate. Each map corresponds to an area on the substrate of $100 \times 100 \mu \mathrm{m}^{2}$ with an excitation wavelength of 633 nm. Substrate was immersed in $1.156 \times 10^{-5} \mathrm{M}(\mathrm{aq})$ RBITC for 15 minutes and then dried in $\mathrm{N}_{2}$ before SERS measurement. 
In addition to BPE, another common SERS probe, rhodamine B isothiocyanate (RBITC) (aq) $\left(1.156 \times 10^{-5} \mathrm{M}\right)$ was used to test the $\mathrm{Cu}$ polymer substrate. Fig. 6 (a) shows SERS spectra of RBITC on CuPoly and CuSi substrates, each averaged from 800 individual spectra collected from mapping, where characteristic peaks match those described in the literature [37].

Integrated peak intensity of the $1200 \mathrm{~cm}^{-1}$ band for the CuPoly substrate is about 3 times that of its CuSi counterpart along with a good reproducibility of $11.0 \%$ as observed from SERS mapping, fig. 6(b), (c). It is worth pointing out that although the improvement in SERS performance in the case of copper is not as significant as that of silver, the possibility of manufacturing nanorod arrays with designed gaps and diameters using this guided OAD method should allow further structural optimization. This added to the low cost of polymer substrates makes this novel method an attractive approach for fabricating large-scale, highly effective SERS active substrates.

Another $\mathrm{Cu}$ nanorod polymer array of rod length $989 \mathrm{~nm}$ was manufactured, with the height of the $1200 \mathrm{~cm}^{-1}$ band averaged from 20 BPE spectra. This array produces a SERS signal strength about twice that of the Cu-glass control, and about half that of the $671 \mathrm{~nm}$ polymer array, with a RSD of $11.6 \%$. The magnitude of the normalized electric field of $\mathrm{Cu}$ nanorod arrays of both lengths was simulated using DDA. The results for which are illustrated in figure 7 . The average enhancement factors of $671 \mathrm{~nm}$ and $989 \mathrm{~nm}$ arrays were found to be $\underline{83.5 \text { and } 52.7 \text { respectively, which is consistent with experimental results. This suggests that }}$ $\underline{\text { SERS intensity is not simply proportional to rod surface area, and that aspect ratio changes }}$ which give control over the longitudinal and transverse plasmons play an important role. Therefore it is possible to optimize rod length for the best SERS effect. 

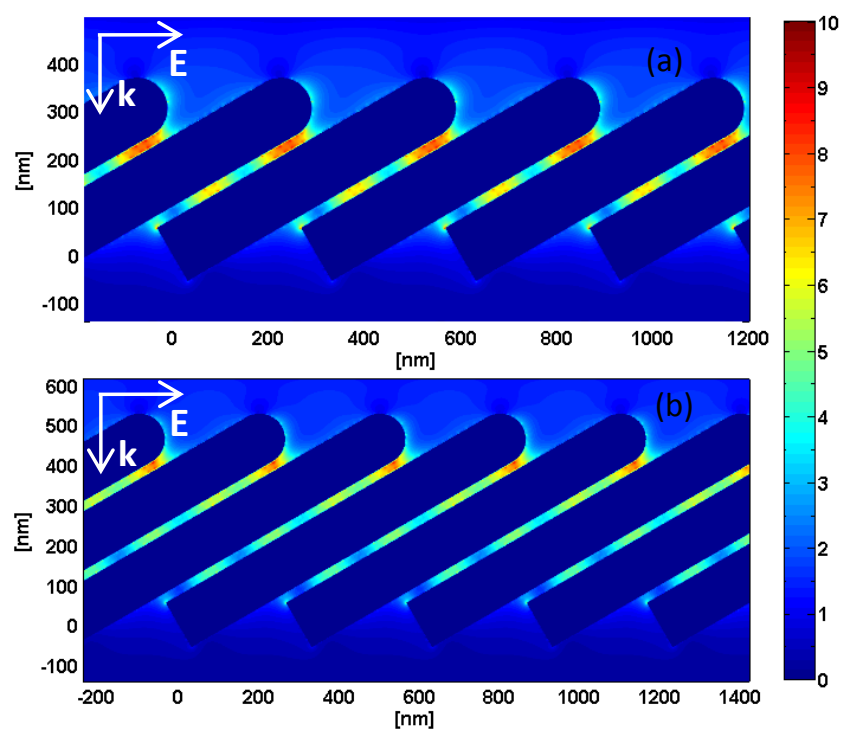

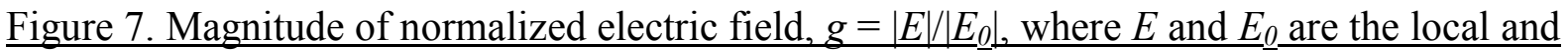
incident fields respectively, of $\mathrm{Cu}$ nanorod arrays of (a) $671 \mathrm{~nm}$ and (b) $989 \mathrm{~nm}$ length. Diameter $\sim 127 \mathrm{~nm}$; tilted angle $\sim 30^{\circ}$, as taken from SEM measurements. The dielectric constants of $\mathrm{Cu}$ were from Johnson and Christy [29]. All internal fields were set to zero for clarity.

\section{Conclusion}

In summary, we have demonstrated a proof-of-principle fabrication method for highly ordered nanorod arrays which, in principle, could circumvent the problems of gap-size and diameter control. The polymer template tightly controls the nucleation process during OAD, producing nanorod arrays which are highly SERS-active and reproducible. In the case of Ag, an enhanced SERS intensity about 10 times that of the Ag-silica control was observed for the $85^{0}$ Ag-polymer array. The SERS response of Ag-polymer arrays was compared to results obtained from discrete dipole approximation simulations, which revealed that narrow gaps between nanorods, formed by this guided nucleation approach, were responsible for this dramatic enhancement. Ordered Cu-polymer arrays were also successfully fabricated, which 
likewise, exhibited strong SERS combined with good reproducibility, as confirmed via mapping studies of BPE and RBITC. Although we employ only one nano-pattern in this experiment the results highlight that potentially, such templates could be created to desired specifications before rod deposition. Future studies could focus on optimisation of polymer template design, and in turn, plasmonic characteristics to maximise SERS performance. This work opens the door not only to an efficient, cheap and reproducible method of producing high-quality, high-order nanorod-arrays as SERS substrates, but to their wider application in other areas such as nanophotonic devices and solar cells.

\section{Acknowledgement}

The authors thank Dr. S. Mabbott for his assistance in experimental work. M. Keating acknowledges the financial support of an EPSRC Doctoral Training Grant. G. Wei thanks China Scholarship Council (CSC) for the financial support. Simulation results were obtained using the EPSRC funded ARCHIE-WeSt High Performance Computer (www.archiewest.ac.uk). EPSRC grant no. EP/K000586/1.

Supporting Information Available: Further experimental details are included as well as SEM images of: bare polymer, $0^{0} \mathrm{Ag}$ on polymer, and $85^{\circ} \mathrm{Ag}$ on $\mathrm{Si}$. Included also are 2-D Ag nanorod surface areas as measured from SEM, SERS spectra, and simulation structures This information is available free of charge via the internet at http://pubs.acs.org 
References

1. Willets, K. A.; Van Duyne, R. P.; Ann. Rev. Phys. Chem. 2008, 58, 267-297

2. Li, J. F.; Huang, Y. F.; Ding, Y.; Yang, Z. L.; Li, S. B.; Zhou, X. S.; Fan, F. R.; Zhang, W.; Zhou, Z. Y.; Wu, D. Y.; Ren, B.; Wang, Z. L.; Tian, Z. Q. Nature, 2010, $464,392-395$

3. Camden, J. P.; Dieringer, J. A.; Wang, Y. M.; Masiello, D. J.; Marks, L. D.; Schatz, G. C.; Van Duyne, R. P. J. Am. Chem. Soc. 2008, 130,12626

4. Graham, D.; Thompson, D. G.; Smith, W. E.; Faulds, K. Nat Nanotechnol. 2008, $3(9), 548-551$

5. Sisco, P. N.; Murphy, C. J. J Phys. Chem. A 2009, 113(16) 3973-3978

6. Mahmoud, M. A.; Tabor, C. E.; El-Sayed, M. A. J Phys. Chem. C 2009, 113(14) $5493-5501$

7. Keating, M.; Chen, Y.; Lamour, I. A.; Faulds, K.; Graham, D. Meas. Sci. Technol. 2012, 23(8) $084006[10]$

8. Driskell, J. D.; Shanmukh, S.; Liu, Y.; Chaney, S. B.; Tang, X. J.; Zhao, Y. P.; Dluhy, R. A. J. Phys. Chem. C 2008, 112, 895-901.

9. Liu Y.J.; Zhang Z.Y.; Zhao Q.; Dluhy R. A; Zhao Y.P., J. Phys. Chem. C 2009, 113, 9664-9669.

10. Liu Y.; Chu H.; Zhao Y. J. Phys. Chem. C 2010, 114, 8176-8183.

11. Song, C.; Abell, J. L; He, Y.; Hunyadi Murph, S.; Cui, Y.; and Zhao, Y. J. Mater. Chem. 2012, 22, 1150

12. Plaza, J. L.; Chen, Y.; Jacke, S.; Palmer, R. E. Langmuir, 2005, 21, 1556

13. Driskell, J. D.; Shanmukh, S.; Liu, Y.-J; Hennigan, S.; Jones, L.; Zhao, Y.-P.; Dluhy, R. A.; Krause, D. C.; Tripp, R. A. IEEE Sensors Journal 2008, 8, 863-870 
14. Liu, Y.-J.; Zhang, Z.-Y.; Dluhy, R. A.; Zhao, Y.-P. J. Raman Spectrosc. 2010, 41, $1112-1118$

15. Boltasseva, A.; J. Optics A 2009, 11, 114001

16. Wu, W.; Hu, M.; Ou, F. S.; Li, Z.; Williams, R. S. Nanotechnology 2010, 21, 255502

17. Alvarez-Puebla, R.; Cui, B.; Bravo-Vasquez, J.-P.; Veres, T.; Fenniri, H. J. Phys. Chem. C 2007, 111, 6720-6723

18. Singh J.P.; Chu H; Abell J.; Tripp R.A.; Zhao Y. Nanoscale 2012, 4, 3410-4

19. Song, S.; Keating, M.; Chen, Y.; Placido, F. Meas. Sci. Technol. 23, 2012, 084007

20. Chaney, S. B.; Shanmukh, S.; Dluhy, R. A.; Zhao, Y.-P. App. Phys. Lett. 2005, 87, 031908

21. Geissler, M.; Li, K.; Cui, B.; Clime, L.; Veres, T. J. Phys. Chem. C 2009, 113, $17296-17300$

22. Liu, Y.; Fan, J.; Zhao, Y.P.; Shanmukh, S.; Dluhy, R. A._Appl. Phys. Lett. 89, 173134 (2006); doi: 10.1063/1.2369644

23. Leverette, C. L.; Jacobs, S. A.; Shanmukh, S.; Chaney, S. B.; Dluhy, R.A.; Zhao, Y.P. Appl. Spectrosc., 200660 906-13

24. Keskin S.; Kahraman, M.; Culha M. Analyt. Chem. 2010, 82, 7596-7602

25. Draine, B. T.; Flatau, P. J. J. Opt. Soc. Am. A 1994, 11, 1491-1499

26. Draine, B. T.; Flatau, P. J. J. Opt. Soc. Am. A 2008, 25, 2693

27. Flatau, P. J.; Draine, B. T. Opt. Exp. 2012, 20, 1247-1252.

28. Draine, B. T.; Flatau, P. J. User Guide for the Discrete Dipole Approximation Code DDSCAT 7.2 http://arxiv.org/abs/1202.3424 (accessed Mar 18, 2013).

29. Johnson, P. B.; Christy, R. W. Phys. Rev. B 1972, 6, 4370-4379 
30. Li, S.; Pedano, M. L.; Chang, S.-H.; Mirkin, C. a; Schatz, G. C. Gap structure effects on surface-enhanced Raman scattering intensities for gold gapped rods. Nano letters 2010, 10, 1722-1727.

31. Muniz-Miranda M.; Gellini C.; Giorgetti E. J Phys. Chem. C 2011, 115, 5021-5027.

32. Kong C.; Sun S.; Zhang X.; Song X.; Yang Z. CrystEngComm 2013, 15, 6136-6139.

33. Shao Q.; Que R.H.; Shao M. W.; Cheng L.; Lee S.T. Adv. Funct. Mater. 2012, 22,2067-2070.

34. Xu D. P.; Dong Z. M.; Sun J. L. Mater. Lett. 2013, 92, 143-146

35. Zhao A.; Mei T.; Lin X.; Ni L.; Wu A. Proc. Int. Conf. Inf. Acq. 2007 1-4244-1220.

36. Khan M. A.; Hogan T. P.; Shanker B. J Nano Syst. Technol. 2009, 1, 1

37. Fang C.; Agarwal A.; Buddjaraju K. D.; Khalid N. M.; Salim S. M.; Widjaja E.; Garland M. V., Balasubramanian N.; Kwong D. L. Biosen. Bioelectro. 2008, 24 216221. 